\title{
Recurrent Point Processes with Dependent Interference with Reference to Neuronal Spike Trains
}

\author{
M, TEN HOOPEN AND H. A. REUVER \\ Institute of Medical Physics TNO, Utrecht, The Netherlands \\ Communicated by Richard Bellman
}

\begin{abstract}
The interaction between a recurrent point process and another point process, generated directly or indirectly by the former, is treated as an extension of the interaction of two independent recurrent processes. The model originated from, and is thought applicable to, the statistical analysis of nerve cell discharge patterns. Applications of the theory to optimization problems in other fields are considered.
\end{abstract}

\section{INTRODUCTION}

In previous papers [1, 2] selective interaction between two independent recurrent time sequences of stimuli, called excitatory and inhibitory, was considered. The intervals between the stimuli in each sequence were independent of each other and identically distributed with probability density functions $\phi(t)$ and $\psi(t)$, respectively. The following mode of interaction was operative. An inhibitory stimulus annihilated the next excitatory stimulus. If there were two or more inhibitory stimuli without an interjacent excitatory stimulus, only one subsequent excitalory stimulus was deleted. Every excitatory stimulus provoked a response, provided it had not been deleted by an immediately preceding inhibitory stimulus. The probability density function $p(t)$ of the thus-transformed excitatory process was derived.

The problem originated from a characteristic of certain neural discharge patterns, the stimuli being identified with nervous impulses. In this 
application (among others), however, the assumption of the independence of the two channels is open to doubt. The present communication is intended as an addendum that abandons this supposition.

For instance, it is fairly well established that inhibitory interference with excitation occurs in the spinal cord by a local feedback path, and that branches from the motor axon run back into the ventral horn, exciting internuncial Renshaw cells, which in turn inhibit the neighboring motoneurons [3]. Some authors $[4,5]$ have suggested that inhibitory action may be some form of general damping of motoneuron excitability. A more specific effect has been proposed by Brooks and Wilson [6], who compared Renshaw cell inhibition to surround inhibition found in sensory systems $[7,8]$. (Reformulated in mathematical terms, this concept of feedback inhibition by a response will be pursued as model II.)

In the theory of visual and acoustical contrast-sharpening phenomena $[9,10]$ besides feedback inhibition, an alternative construction has been envisaged: inhibition that is fed forward, or afferent collateral inhibition. In this case not the response, but the incoming stimuli themselves exert, via internuncials, inhibitory action upon the neurons to be excitated. This mode of interaction is incorporated in model I. In the Discussion we will touch upon possible applications to other fields.

DEFINITIONS AND NOTATION

Let the excitatory process be a recurrent point process called the $\phi$ process with probability density function $\phi(t)$ of the intervals between successive $\phi$ events. The $\phi$ process triggers, in a way specified later, an inhibitory point process called the $\psi$ process. Every $\psi$ event deletes the next $\phi$ event. Every undeleted $\phi$ event is called a response or $p$ event. The probability density function $p(t)$ of the intervals between successive $p$ events is required.

In model I every $\phi$ event, deleted or not, triggers a sequence of $\psi$ events such that the interval between that $\phi$ event and the next $\psi$ event, as well as the intervals between successive $\psi$ events, are independent random variables with the same probability density function $\psi(t)$. The sequence continues until the next $\phi$ event, deleted or not, occurs; this $\phi$ event in turn triggers a new sequence of $\psi$ events. In model II only undeleted $\phi$ events (responses) have the described trigger effect.

Mathenatical Biosciences 2, $1-10(1968)$ 
The terms $\bar{t}_{\phi}, \bar{t}_{\psi}$, and $\omega(t)$ are defined by

$$
\begin{gathered}
\bar{t}_{\phi}=\int_{i}^{\infty} t \cdot \phi(t) \cdot d t, \\
\bar{t}_{\psi}=\int_{i}^{\infty} t \cdot \psi(t) \cdot d t, \quad \text { and } \quad \omega(t)=\int_{i}^{\infty} \psi(v) d v .
\end{gathered}
$$

By way of exercise we will insert a Poisson process for the excitatory process. It will be noted that insertion of an exponential function for $\psi(t)$ leads to the equivalent of case A of [1]. Consequently, that case can be considered as a special form of model I or II. In these special applications the solution for $p(t)$ can be expressed in a compact form by introducing the Laplace transform method, the Laplace transform of a function being designated by the corresponding capital with argument $s$, for example, $P(s)$ for $p(t)$. From $P(s)$ the mean interval $\bar{t}$ and the standard deviation $\sigma$ of the probability density function $p(t)$ can be obtained by differentiation.

MONEL I

To derive $p(t)$ we introduce the auxiliary functions $\theta_{k}(t)$ and $\pi_{k}(t)$. $\eta_{k}(t) \cdot d t$ denotes the probability that the interval between the last $p$ event and the $k$ th $\phi$ event after that $p$ event is in $(t, t+d t)$ while that $k$ th $\phi$ event is not a $p$ event. Thus

$$
\theta_{1}(t)=\phi(t) \int_{i}^{t} \psi(v) d v
$$

Since the $\psi$ events are triggered by the $\phi$ events, $\theta_{k}(t)$ is the $k$-fold convolution of $\theta_{\mathbf{1}}(t)$ given by the recurrence relations

$$
\theta_{k}(t)=\int_{i}^{t} \theta_{k-1}(v) \theta_{1}(t-v) d v \quad \text { for } \quad k \geqslant 2
$$

$\pi_{k}(t) \cdot d t$ denotes the probability that the interval between two successive $p$ cvents is in $(t, t-d t)$ while $k \phi$ events have occurred in between. Therefore, 


$$
\pi_{0}(t)=\phi(t) \int_{i}^{\infty} \psi(v) d v=\phi(t) \omega(t)
$$

$\pi_{k}(t) \cdot d t$ is equal to the integral of the product of the probabilities that:

(a) the interval between the last $p$ event and the $k$ th $\phi$ event after that $p$ event is in $(v, v+d v)$ with $0<v<t$;

(b) the interval between that $k$ th $\phi$ event and the next $\phi$ event is in $(t-v, t-v+d t)$; and

(c) the interval between that $k$ th $\phi$ event and the next $\psi$ event is greater than $t-v$.

Since the $\psi$ events are triggered by the $\phi$ events, it follows that

$$
\begin{aligned}
\pi_{k}(t) & =\int_{0}^{t} \theta_{k}(v) \phi(t-v)\left[\int_{t=v}^{\infty} \psi(w) d w\right] d v \\
& =\int_{0}^{t} \theta_{k}(v) \pi_{0}(t-v) d v .
\end{aligned}
$$

Finally,

$$
p(t)=\sum_{k=0}^{\infty} \pi_{k}(t)
$$

For $\phi(t)=\mu \exp (-\mu t)$ we have

$$
\Theta_{\mathbf{1}}(t)=\mu \exp (-\mu t) \int_{0}^{t} \psi(v) d v
$$

and

$$
\tau_{0}(t)=\mu \exp (-\mu t) \int_{i}^{\infty} \psi(v) d v
$$

Taking the Laplace transform, we have

$$
\begin{aligned}
& \Theta_{1}(s)=\mu(s+\mu)^{-1} \Psi(s+\mu) \\
& \Pi_{0}(s)=\mu(s+\mu)^{-1}[1-\Psi(s+\mu)] .
\end{aligned}
$$

Mathematical Biosciences 2, 1-10 (1968) 
Since $\theta_{k}(t)$ is the $k$-fold convolution of $\theta_{1}(t)$, we have $\theta_{k}(s)=\theta_{1}^{k}(s)$. While $\pi_{k}(t)$ is the convolution of $\theta_{k}(t)$ and $\pi_{0}(t)$, it follows that

$$
\Pi_{k}(s)=\theta_{k}(s) \cdot I_{0}(s)
$$

Finally,

$$
P(s)=\sum_{k=0}^{k} H_{k}(s)
$$

Elaboration yields

$$
P(s)=\mu[1-\Psi(s+\mu)][s+\mu-\mu \Psi(s+\mu)]^{-1} .
$$

MONKL H

We introduce the auxiliary functions $\chi_{k}(t, \tau)$ and $p_{k}(t), \chi_{k}(t, \tau) \cdot d t \cdot d \tau$ denotes the probability that the interval between the last $p$ event and the $k$ th $\psi$ event after that $p$ event is in $(\tau, \tau+d \tau)$ and that the interval between the last $p$ event and the $\phi$ event next to that $k$ th $\psi$ event is in $(t, t+d t)$. Then,

$$
\chi_{1}(t, \tau)=\phi(t) \psi(\tau) \quad \text { for } \quad t>\tau
$$

and zero elsewhere.

To compute $\chi_{k}(t, \tau)$ for $k \geqslant 2$ we note that $\chi_{k}(t, \tau) \cdot a t \cdot d \tau$ equals the sum of:

(1) the integral of the product of the probabilities that

(a) the interval between the last $p$ event and the $(k-1)$ th $\psi$ event after that $p$ event is in $(w$, $w+d w)$ while the interval between the last $p$ event and the $\phi$ event next to that $(k-1)$ th $\psi$ event is $(v, v+d v)$ with $0<w<v<\tau$;

(b) the interval between that $(k-1)$ th $\psi$ event and the next $\psi$ event is in $(\tau-w, \tau-w+d \tau)$; and

(c) the interval between the $\phi$ event mentioned in (a) and the $\phi$ event next to it is in $(t-v, t-v+d t)$.

(2) the integral of the product of the probabilities that

(a) the interval between the last $p$ event and the $(k-1)$ th $\psi$ event after that $p$ event is in $(w, w+d w)$ while the interval between the 
last $p$ event and the $\phi$ event next to that $(k-1)$ th $\psi$ event is in $(t, t+d t)$ with $0<w<\tau$; and

(b) the interval between that $(k-1)$ th $\psi$ event and the next $\psi$ event is in $(\tau-w, \tau-w+d \tau)$.

It follows that

$$
\begin{aligned}
\chi_{k}(t, \tau)= & \iint_{\tau=\tau>>>0} \phi(t-v) \psi(\tau-\tau) \chi_{k-1}(v, w) d v d \tau \\
& +\int_{0}^{\tau} \psi(\tau-w) \chi_{k-1}(t, w) d w .
\end{aligned}
$$

The expression $p_{k}(t) \cdot d t$ denotes the probability that the interval between two successive $p$ events is in $(t, t+d t)$ while $k \psi$ events have occurred in between. Then, since the $\psi$ events are triggered by the $p$ events,

$$
p_{0}(t)=\phi(t) \cdot \omega(t) \text {. }
$$

To compute $p_{k}(t)$ for $k \geqslant 1$, we note that $p_{k}(t) \cdot d t$ equals the integral of the product of the probabilities that:

(a) the interval between the last $p$ event and the $k$ th $\psi$ event after that $p$ event is in $(w, w+d w)$ while the interval between the last $p$ event and the $\phi$ event next to that $k$ th $\psi$ event is in $(v, v+d v)$ with $0<w<$ $v<t$;

(b) the interval between that $k$ th $\psi$ event and the next $\psi$ event is greater than $t-w$; and

(c) the interval between the $\phi$ event mentioned in (a) and the $\phi$ event next to it is in $(t-v, t-v+d t)$.

Therefore,

$$
p_{k}(t)=\iint_{t>v^{\prime}=0} \omega(t-w) \phi(t-v) \chi_{k}(v, w) d v d w .
$$

Finally,

$$
p(t)=\sum_{k=0}^{\infty} p_{k}(t)
$$


For $\phi(t)=\mu \exp (-\mu t)$ we have

$$
\chi_{1}(t, \tau)=\psi(\tau) \mu \exp (-\mu t)
$$

for $t>\tau$ and zero elsewhere.

$$
\begin{aligned}
\chi_{k}(t, \tau)= & \exp (-\mu t) \int_{t>\tau>\bigcup_{>-w^{\prime}>0}} \mu \exp (\mu v) \psi(\tau-w) \chi_{k-1}(\tau, w) d v d w \\
& +\int_{0}^{\tau} \psi(\tau-w) \chi_{k-1}(t, w) d w .
\end{aligned}
$$

Or,

$$
\begin{aligned}
\exp (\mu t) \chi_{k}(t, \tau)= & \int_{t>\tau>v} \mu \exp (\mu v) \psi(\tau-w) \chi_{k-1}(v, w) d v d w \\
& +\int_{0}^{\tau} \psi(\tau-\tau) \exp (\mu t) \chi_{k-1}(t, w) d w
\end{aligned}
$$

While $\partial / \partial t\left[\exp (\mu t) \chi_{1}(t, \tau)\right]=0$, it follows by induction that

$$
\frac{\partial}{\partial t}\left[\exp (\mu t) \chi_{k}(t, \tau)\right]=0 \quad \text { for } \quad k \geqslant 2
$$

Therefore, $\exp (\mu t) \chi_{k}(t, \tau)$ is a function of $\tau$ only, denoted by $\chi_{k}(\tau)$.

We have

$$
\begin{aligned}
& \chi_{1}(\tau)=\mu \psi(\tau) \\
& \chi_{k}(\tau)=\mu \int_{0}^{\tau} \psi(\tau-w)(\tau-w) \chi_{k-1}(w) d w+\int_{i}^{\tau} \psi(\tau-w) \chi_{k-1}(w) d w .
\end{aligned}
$$

Taking the Laplace transform yields

$$
\begin{aligned}
& X_{1}(s)=\mu \Psi(s) \\
& X_{k}(s)=[\Psi(s)-\mu \Psi(s)] X_{k-1}(s) \quad \text { with } \quad \dot{\Psi}(s)=\frac{d}{d s} \Psi(s)
\end{aligned}
$$


Then,

$$
X_{k}(s)=\mu \Psi(s)[\Psi(s)-\mu \dot{\Psi}(s)]^{k-1}
$$

Further,

$$
\begin{aligned}
& p_{0}(t)=\mu \exp (-\mu t) \omega(t), \\
& p_{k}(t)=\int_{0}^{t} \omega(t-w) \mu \exp (-\mu t)(t-w) \chi_{k}(w) d w .
\end{aligned}
$$

Taking the Laplace transform gives

$$
\begin{aligned}
& P_{0}(s)=\mu(s+\mu)^{-1}[1-\Psi(s+\mu)], \\
& P_{k}(s)=\mu(s+\mu)^{-2}[1-\Psi(s+\mu)+(s+\mu) \dot{\Psi}(s+\mu)] X_{k}(s+\mu), \\
& P(s)=\sum_{k=0}^{\infty} P_{k}(s) .
\end{aligned}
$$

Elaboration yields

$$
\begin{aligned}
P(s)= & \mu(s+\mu)^{-1}+\mu s(s+\mu)^{-2} Y^{\prime}(s+\mu) \\
& \times[\Psi(s+\mu)-1][1-\Psi(s+\mu)+\mu \dot{\Psi}(s+\mu)]^{-1} .
\end{aligned}
$$

\section{DISCUSSION}

A model based on the selective interaction between two independent time sequences and treated previously $[1,2]$ has led to a particular characterization of multimodal and long-tailed impulse interval distributions of single nerve cells [11]. The two models dealt with in the present paper are based on the interaction between nonindependent time sequences. They were introduced as a natural extension of the former model. It is hoped that they, too, will be of help in interpreting neuronal discharge patterns. The following are examples of possible future applications.

The conditions in which models give rise to a more or less constant output frequency when the input frequency changes might be examined. (In other words, the arrangement of connections that stabilizes the output 
could be determined.) As mentioned in the Introduction, this question is of interest in relation to the role of the Renshaw cells in the spinal cord in modulating molor control. Conversely, the circunstances in which a steeply increasing output frequency will result upon gradually increasing the input frequency might be investigated. This thresholdlike property is related to the tendency toward analogue-binary conversion of signals in the nervous system as noticed by Uttley [12] and Whitfield [13].

In addition, with the mode of interaction just defined in mind, it is conceivable that there exist applications to problems in quite different fields. The stimuli dealt with need not be nervous impulses; they may be taken as unitary events in a general sense. A stream of events $\phi$ that are to arrive at a certain point or goal but are distorted by other events $\psi$ could be imagined. This distortion may represent an intentional obstruction or may be caused by a neutral source. As such, it recalls a comparison with hit and antihit problems in game theory, prey-andpredator systems in ecology, and psychophysical rivalry situations. We will only touch upon these applications and adopt the nomenclature of an attacker $\phi$ and a defender $\psi$.

For $\phi, \mathbf{1} / \bar{t}$ is a measure of success for coming through a barrier: $\bar{t}_{\phi} / \bar{t}$ equals the proportion of hits. For $\psi, \bar{t}$ is a measure for repulsing the attack. Given the attacker's stratcgy, characterizcd by $\phi(t)$, one may ask which probability density function $\psi(t)$ maximizes $\bar{t}$, with certain constraints, for instance, a given $\tilde{t}_{\psi}$ that measures the effort that $\psi$ is capable, or willing, to bring in for the defense. Vice versa, given $\psi(t)$, which $\phi(t)$ minimizes $\bar{t}$ (again possibly for given $\bar{t}_{\phi}$ )? Model I reflects the situation where the defense is alert and reacts to every attack, whereas in model II the defense comes into action only after a hit has occurred. If, for a given $\phi(t)$, the two models are compared with regard to maximizing $\bar{t}$, interesting problems come to light, particularly if a delay in response to an attack and to a hit, respectively, are introduced.

A general approach to these problems consists of optimizing the probability density function being sought by the method of dynamic programming $[14,15,16]$ or of approximating the required function by a convex linear combination of suitable positive unit area functions and optimizing the coefficients by a modified Lagrangian multiplier technique [17]. When the unknown probability density function belongs to a parametrized family of distributions, the parameters may be adjusted by the same techniques. 


\section{REFERENCES}

1 M. ten Hoopen and H. A. Reuver, J. Appl. Prob. 2(1965), 286.

2 M. ten Hoopen and H. A. Reuver, Inform. Control 10(1967), 149.

3 P. H. Hammond, P. A. Merton, and G. G. Sutton, Brit. Med. Bull. 12(1956), 214.

4 J. C. Fccles, P. Fatt, and K. Koketsu, J. Physiol. 126(1954), 524.

5 R. Granit, J. E. Pascoe, and G. Steg, J. Physiol. 138(1957), 381.

6 V. B. Brooks and V. J. Wilson, J. Physiol. 146(1959), 380.

7 H. K. Hartline, H. G. Wagner, and F. Ratliff, J. Gen. Physiol. 39(1956), 651.

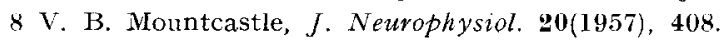

9 J. C. Bliss and W. B. Macurdy, J. Opt. Soc. Amer. 51(1961), 1373.

10 G. G. Furman and L. S. Frishkopf, J. Acoust. Soc. Amer. 36(1964), 2194.

$11 \mathrm{M}$. ten Hoopen, Kybernetik $\mathbf{3}(\mathbf{1 9 6 6 )}, 17$.

I2 A. M. Uttley, EEG Clin. Neurophysiol. 6(1954), 479.

13 I. C. Whitfield, Proc. XXIII Internat. Congr. Physiol. Sci. (Tokyo) IV, 245-24T (Internat. Congr. Ser. No. 87). Excerpta Medica Foundation, Amsterdam.

14 R. E. Bellman, Dynamic programming, Princeton Univ. Press, Princeton, New Jersey, 1957.

15 R. E. Bellman and S. Dreyfus, Applied dynamic programming, Princeton Univ. Press, Princeton, New Jersey, 1962.

16 S. Dreyfus, Dynamic programming and the calculus of variations, Academic Press, New York, 1965.

17 C. W. Churchman, R. L. Ackhoff, and E. L. Arnoff, Introduction to operations research, Wiley, New York, 1957. 